\title{
Electron Energy-loss Spectroscopy Using MerlinEM - Medipix3 Detector
}

Marcel Tencé ${ }^{1}$, Jean-Denis Blazit ${ }^{1}$, Xiaoyan Li $^{1}$, Matus Krajnak ${ }^{2}$, Eduardo Nebot del Busto ${ }^{2}$, Richard Skogeby $^{2}$, Léo Cambou ${ }^{1}$, Mathieu Kociak ${ }^{1}$, Odile Stephan ${ }^{1}$ and Alexandre Gloter ${ }^{1}$

${ }^{1}$ Université Paris-Saclay, CNRS, Orsay, Ile-de-France, France, ${ }^{2}$ Quanum Detectors Ltd., Harwell Oxford, England, United Kingdom

Here, the hybrid pixel detector, Medipix3, has been used for a direct electron detection in the case of EELS spectroscopy. Such detectors have already been used for imaging and diffraction in TEM [1] and STEM [2]. Several advantageous features are expected in the case of EELS (high sensitivity and dynamical ranges, adaptability from 30 to $300 \mathrm{keV}$ electrons and zero read-out noise).

In order to be used as an EELS detector, Quantum Detectors designed a 4x1 MerlinEM detector where 4 Medipix 3 chips are aligned in $4 \times 1$ configuration, resulting in a 1024x 256 detector. A dedicated flange was design to attach the detector to a GATAN ENFINA spectrometer and this EELS system is mounted on a Cs corrected STEM (Nion USTEM200) operated at 60 and $100 \mathrm{keV}$.

When an electron (with an Eo energy) enters in the $500 \mu \mathrm{m}$ thick silicon layer of the Medipix3, it deposits its energy and might thus be detected by a single or several neighbouring pixels. To do so, the deposited energy must exceed the pre-set lower threshold energy value, $\mathrm{TH}$, and then a count is registered in the corresponding pixel circuitry. By setting a high threshold energy, most of the electrons can only give enough energy to be detected on a single pixel (or even be undetected), resulting in a narrow point spread function (PSF). By decreasing the threshold energy, a single electron can deposit enough energy on several pixels leading to several counts and an higher detector quantum efficiency (DQE). Additionally, the detector might work in charge summing mode (CSM), where the induced current of the pixel is compared with the induced current of the surrounding pixels. Only the pixel with the highest current is thus credited of the count. This reduces the impact of charge sharing on the PSF whilst maintaining the high DQE of low threshold energies. As a result, there are several options to balance between optimizing the DQE or the PSF, that might be adapted for different EELS applications (core-loss, bang gap measurement, ...) or for different electron energies Eo. Figure 1a shows two zero-loss peaks (ZLP) measured at different TH at $\mathrm{Eo}=60 \mathrm{keV}$ and $\mathrm{Eo}=100 \mathrm{keV}$. They have been collected with an energy dispersion of ca. $1.5 \mathrm{eV} / \mathrm{ch}$ and confirm that the PSF get better at lower Eo and at higher TH. Nevertheless, even at $100 \mathrm{keV}$ and at $\mathrm{TH}=$ $15 \mathrm{keV}$, the PSF is of high quality with $63 \%$ of the ZLP intensity in a single pixel. Due to the high DQE, it is possible to perform spectrum imaging with atomic resolution in the range of several $\mathrm{keV}$ loss. The figure $1 b$ show the chemical map obtained on a $\mathrm{LaMnO}_{3}$ (LMO) thin film grown on $\mathrm{SrTiO}_{3}$ (STO) [3] using a $250 \mathrm{pA}$ probe at $100 \mathrm{keV}$. The Ti and La atomic columns have been mapped through the Ti-K $(4.9 \mathrm{keV})$ and La-L (5.5keV) edges collected with $10 \mathrm{~ms}$ acquisition time per pixel. The maps are obtained by traditional edge subtraction on the raw data. It is also to note the advantage of "high" energy loss practiced with a rather low voltage $\mathrm{Eo}=100 \mathrm{keV}$ where beam damages are limited. For instance, it enables the use of larger electron doses (250pA for several minutes) for the measurement of fine structures of transition metal (TM)-K edges pre-peaks, that could be relevant to probe the local electronic structure in complement to the more established TM-L2,3 EELS edges studies (Fig 1b).

Another appealing feature of the Medipix3 is the very large dynamic range that derives from a 24-bit maximum counter depth. Every pixel can accumulate $2^{\wedge} 24=16.8 \mathrm{e} 6$ counts before saturating and if the 
EELS spectra is spread over the non-dispersive axis, it means that a billion of counts might be obtained per energy channel. It then becomes possible to measure a non-saturated zero-loss peak, while weak signals from core-losses can be measured thanks to the intrinsic low noise level of counting detectors. Fig 2a,b show such an example for the same $\mathrm{LaMnO}_{3}$ thin film grown on $\mathrm{SrTiO}_{3}$ where the ZLP has been acquired along the core losses up to the La-M. It is thus possible to obtain a wide variety of maps (ZLP, La-N versus La-M, etc...). The difference of spatial resolution (delocalisation) between edges of low and high energy for a given element is clearly visible.

This work has received support from the National Agency for Research under the program of future investment TEMPOS CHROMATEM with the Reference No. ANR-10-EQPX-50.
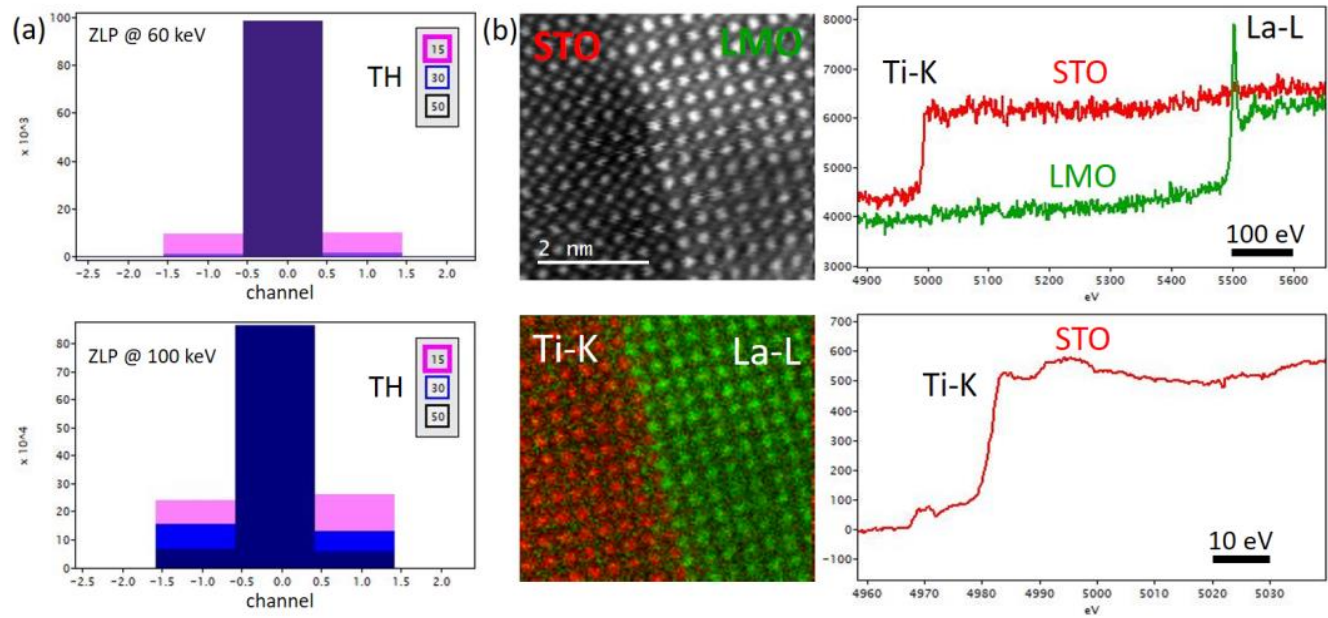

Figure 1. (a) ZLP for different threshold energies TH, (b) HAADF and Ti-K, La-L EELS maps along with the EELS spectra from the STO and LMO sides. In the case of STO, EELS fine structures of the TiK pre-peak (4970 eV) have been measured.

(a)
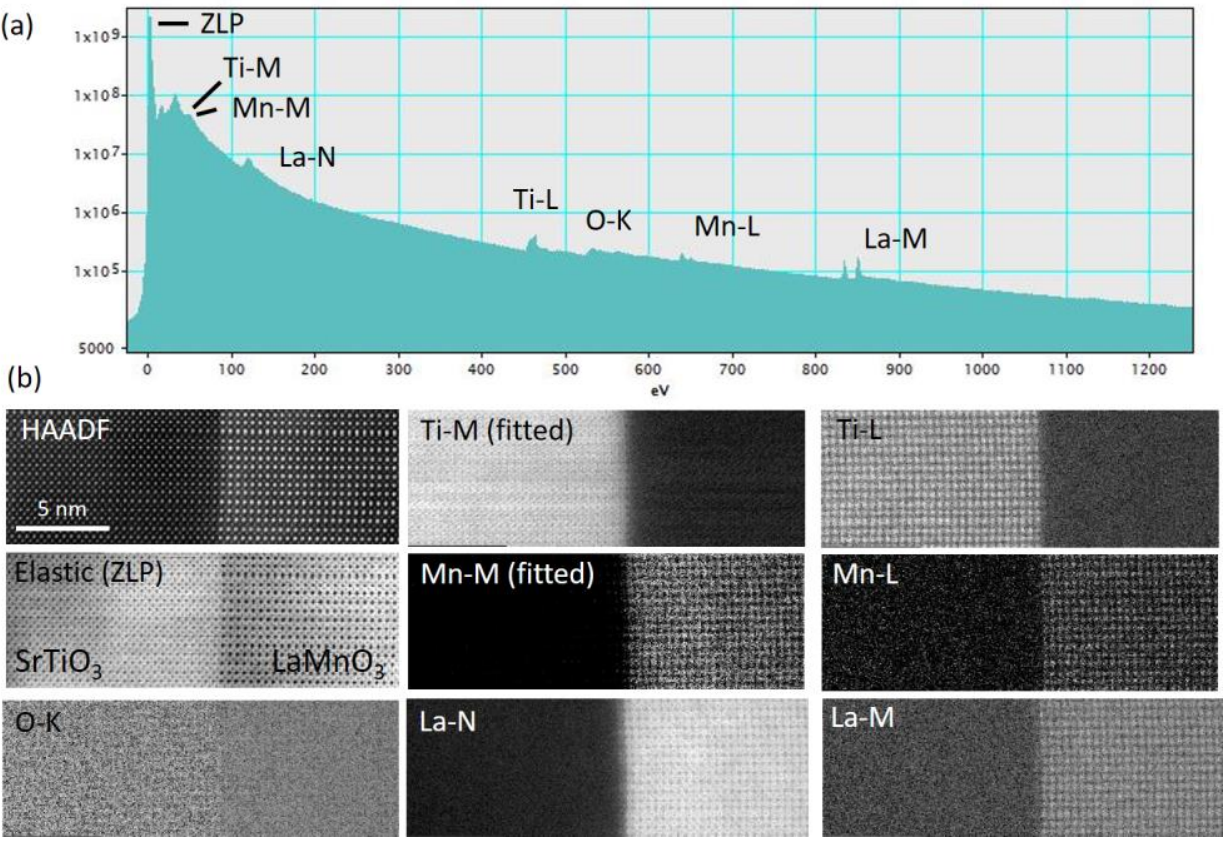
Figure 2. (a) EELS spectrum integrated from the spectrum image showing the different edges observable at the STO/LMO interface, (b) HAADF image and the different maps obtained from the EELS spectrum image.

\section{References}

[1] Mir et al., Characterisation of the Medipix3 detector for 60 and $80 \mathrm{keV}$ electrons, Ultramicroscopy 182 (2017) 44-53.

[2] Krajnak et al., Pixelated detectors and improved efficiency for magnetic imaging in STEM differential phase contrast, Ultramicroscopy 165 (2016) 42-50.

[3] Gibert et al., Interfacial Control of Magnetic Properties at $\mathrm{LaMnO}_{3} / \mathrm{LaNiO}_{3}$ Interfaces, Nano Lett. (2015), 15, 7355-7361. 